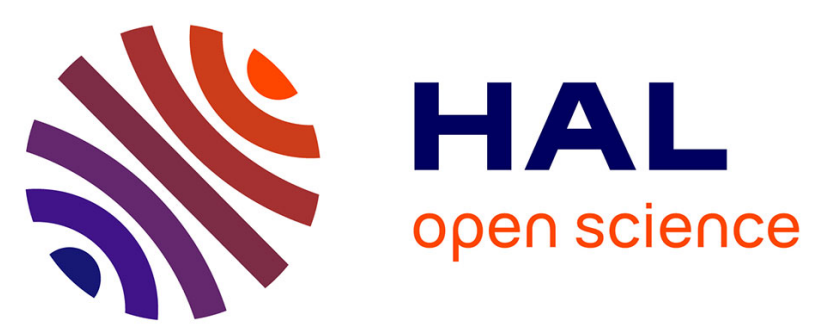

\title{
Motion effect modeling in multipath configuration using warping based lag-doppler filtering
}

Nicolas Josso, Cornel Ioana, Cedric Gervaise, Yann Stéphan, Jerome I. Mars

\section{To cite this version:}

Nicolas Josso, Cornel Ioana, Cedric Gervaise, Yann Stéphan, Jerome I. Mars. Motion effect modeling in multipath configuration using warping based lag-doppler filtering. ICASSP 2009 - IEEE International Conference on Acoustics, Speech and Signal Processing, Apr 2009, Taipei, Taiwan. pp.2301-2304, 10.1109/ICASSP.2009.4960080 . hal-00386481

\section{HAL Id: hal-00386481 \\ https://hal.science/hal-00386481}

Submitted on 22 May 2009

HAL is a multi-disciplinary open access archive for the deposit and dissemination of scientific research documents, whether they are published or not. The documents may come from teaching and research institutions in France or abroad, or from public or private research centers.
L'archive ouverte pluridisciplinaire HAL, est destinée au dépôt et à la diffusion de documents scientifiques de niveau recherche, publiés ou non, émanant des établissements d'enseignement et de recherche français ou étrangers, des laboratoires publics ou privés. 


\title{
MOTION EFFECT MODELING IN MULTIPATH CONFIGURATION USING WARPING BASED LAG-DOPPLER FILTERING
}

\author{
Nicolas F. Josso ${ }^{1}$, Cornel Ioana ${ }^{1}$, Cedric Gervaise ${ }^{2}$, Yann Stephan $^{3}$ and Jérôme I. Mars ${ }^{1}$ \\ ${ }^{1}$ Grenoble INP, GIPSA-lab, 961, rue de la Houille Blanche, 38402 St Martin d'Hères, France \\ [nicolas.josso, cornel.ioana, jerome.mars]@gipsa-lab.inpg.fr \\ ${ }^{2}$ ENSIETA, DTN Lab, 2 rue François Verny, 29806 Brest Cedex, France \\ E-mail : cedric.gervaise@ensieta.fr \\ ${ }^{3}$ Military Center of Oceanography, SHOM, BP 426, 29275 Brest, France \\ E-mail : yann.stephan@shom.fr
}

\begin{abstract}
The estimation of the impulse response (IR) of a propagation channel is necessary for a large number of acoustic applications: underwater communication, detection and localization, etc. Basically, it informs us about the distortions of a transmitted signal in one channel. This operation is usually subject to additional distortions due to the motion of the transmitter-channel-receiver configuration. This paper points on the effects of the motion while estimating the IR by matching filtering between the transmitted and the received signals and introduces a new motion compensation method. Knowing the transmitted signal, the "apparent" speed of each propagation path can be estimated using wideband ambiguity function [1]. Indeed, some interference appears in the wideband ambiguity plane because of the multipath propagation. A warpingbased lag-Doppler filtering method is proposed allowing us to accurately estimate the IR of the channel.
\end{abstract}

Index Terms- Acoustic signal analysis, Motion compensation, Acoustic tomography, Time warping operators.

\section{INTRODUCTION}

Generally, the estimation of the IR of one propagation channel is done with the well-known matching filtering operation [2]. Since the motion of the transmitter-channelreceiver configuration is not known a priori, the received signal could be alternatively correlated with a family of reference signals that represents possible Doppler shifts. The set of reference signals should be defined for all possible velocities and time delays of the configuration [3]. Recently, the Doppler effect consequences on the estimation of the IR in low frequency shallow water environments has been described [1]. The considered processing method in [1] is the matching filtering between the transmitted and received signals. Traditionally, the time delay and Doppler effects are analyzed by the ambiguity function. If the signal is narrow band, the conventional formulation of the ambiguity function is appropriate. In this case, the effects of motion, which are a compression in time for approaching sources and an expansion for receding sources, are approximated as simple carrier-frequency shifts of the transmitted waveform. For the narrow band case, the correlation receiver has a reference set of signals composed by time-delayed and carrierfrequency-shifted versions of the transmitted signal. In our study, a multipath wideband ambiguity function has been introduced in order to take into account the effects of the motion in both the carrier frequency and the modulation function for each path. In the wideband case, the correlation receiver has as reference signals the time-delayed and timecompressed or time-expanded versions of the transmitted one. Interferences may appear in the wideband ambiguity plane because of the multipath propagation which affects the IR estimation. In this paper we introduce a new method for IR estimation in moving configurations, enabling also the classical acoustic inversion processes (ie, defined generally for motionless configurations). This method consists of warping-based lag-Doppler filtering which accurately separates each path in order to reduce interferences and get correct IR estimations. The paper is organized as follows. Section 2 presents the characterization of the multipath environment in a moving configuration. Section 3 describes the warping-based lag-Doppler filtering. The Doppler effect removal technique is described in section 4. Examples on simulated data are presented on section 5 . We conclude in Section 6.

\section{DESCRIPTION OF THE MOTION EFFECT IN A MULTIPATH CONFIGURATION}

In order to describe the motion effect in a multi-path configuration, let us consider a motionless receiver and a transmitter moving with a constant speed. We define a time axis $u$ for the emission (i.e. the time of transmission) and $t$ for the reception (i.e the time of arrival), related in the following way:

$$
u+T_{i}(u)=t,
$$

where $T_{i}(u)$ refers to the time of propagation of the $i^{\text {th }}$ ray. This relation states that a signal transmitted at the time $u$ is received on the $i^{\text {th }}$ ray at the time $u$ plus the propagation time 
along the $i^{\text {th }}$ ray. $L_{i}(u)$ is defined as the length in meters of the $i^{\text {th }}$ ray. With a transmitter moving with a constant speed along axis $x$, (1) becomes

$$
u+\frac{x_{0}+L_{i}(u)+v_{i} u}{c}=t,
$$

where $x_{0}$ is the position of the source when $u=0$ and $c$ is the sound velocity (considered constant). To clarify notations, we introduce

$$
\tau_{i}(u)=\frac{x_{0}+L_{i}(u)}{c} .
$$

Some manipulations with (1), (2) and (3) yields to:

$$
u=\left(t-\tau_{i}(u)\right)\left(\frac{1}{1+v_{i} / c}\right)
$$

The term $\tau_{i}(u)$ represents the time of propagation from the transmitter to the receiver. It depends on the time delay $u$ and cannot be expressed with the time of arrival for all cases. The term $1 /\left(1+v_{i} / c\right)$ is the classical compression or expansion in the time domain induced by the broadband Doppler effect. In equation (4), it has been assumed that Doppler effect depends on each ray. As shown in the figure 1 , the motion vector $v$ is projected on the path of the $i^{\text {th }}$ ray with the declination angle $\theta_{i}$ which leads to

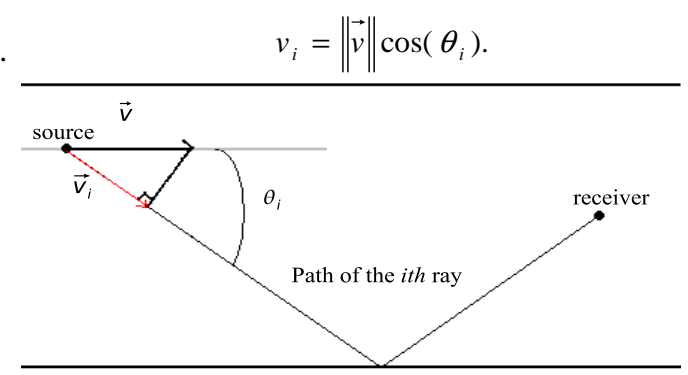

Fig1. Evaluation of vector $v_{i}$ from the motion vector $v$

As indicated by (5), each ray will be characterized by a distinct Doppler effect defined by the speed $v_{\mathrm{i}}$. Considering several rays, the $i^{\text {th }}$ ray is an amplitude-attenuated $\left(a_{i}\right)$, time delayed and Doppler-transformed version of the transmitted signal $e(t)$. With (4) the signal received from the $i^{\text {th }}$ ray is expressed as

$$
s_{i}(t, u)=a_{i}\left(\frac{1}{1+v_{i} / c}\right)^{1 / 2} e\left(\left(t-\tau_{i}(u)\right)\left(\frac{1}{1+v_{i} / c}\right)\right) .
$$

The received signal $s(t, u)$ is the sum of all the $s_{i}(t, u)$ received from each ray leading to the following expression

$$
s(t, u)=\sum_{i} s_{i}(t, u) .
$$

We assume that the propagation time and the projection of the motion vector are constant during transmission. This hypothesis stands valid as long as the distance made by the source during the transmission can be neglected compared with the distance source-receiver. By using the complete formulations of the signal received for each ray and of the apparent speed (5), the formula (7) can be rewritten

$$
s(t)=\sum_{i} a_{i}\left(\frac{1}{1+\|\vec{v}\| \cos \left(\theta_{i}\right) / c}\right)^{1 / 2} e\left(\left(t-\tau_{i}\right)\left(\frac{1}{1+\|\vec{v}\| \cos \left(\theta_{i}\right) / c}\right)\right) .
$$

This expression shows that the Doppler transformation of the transmitted signal $(e(t))$ depends both on the speed of the source and the angle of emission $\left(\theta_{i}\right)$ which is different for each path.

\section{WARPING-BASED LAG-DOPPLER FILTERING}

The problem of the estimation of an IR with matching filtering is formulated and analyzed in this section. It has been explained that the received signal is defined as the sum of amplitude-attenuated, time delayed and Dopplertransformed version of the transmitted signal. Assuming the transmitted signal is known, the propagation time and the velocity associated to each ray can be estimated by cross correlating the received signal with a set of reference signals. The set of reference signals is composed of timedelayed and Doppler-transformed versions of the transmitted signal for all the range of time delays and speeds expected [3]. The factor of contraction or expansion due to the Doppler transformation, $\eta$, satisfies

$$
\eta=\frac{1}{1+v / c} \text {. }
$$

The Doppler transformation factor of the $i^{\text {th }}$ ray is introduced as $\eta_{i}$ and satisfies (9) with the speed $v_{i}$ associated to the same ray. For each reference signal, the cross correlation is expressed as:

$$
R(\tau, v)=\int_{-\infty}^{\infty} s(t+\tau) \eta^{1 / 2} e^{*}(\eta t) d t
$$

where $*$ denotes the complex conjugation, $s(t)$ the received signal and $e(t)$ the transmitted one. Using the expressions of the received signal in a multipath environment (6) and (7) in (10) yields

$$
R(\tau, v)=\sum_{i} a_{i}\left(\eta_{i} \eta\right)^{1 / 2} \int_{-\infty}^{\infty} e\left(\eta_{i}\left(t+\tau-\tau_{i}\right)\right) e^{*}(\eta t) d t .
$$

Local maxima of this correlation function are reached for each ray. For the $i^{\text {th }}$ ray, the maximum is reached when the reference and the propagated signal are exactly aligned in time delay and Doppler. It is assumed that rays are well separated in time in the motionless case so there are no interferences between local maxima. The interferences that could occur between local maxima are studied in [3] and are not the purpose of this paper. The time of propagation and the apparent speed of the $i^{\text {th }}$ ray can be estimated once 
the local maximum is detected. The wideband ambiguity function used here is the square magnitude of (11). This representation can be compared with the classical ambiguity plane except the Doppler effect, modeled as a compression or expansion in time and not as a frequency shifting. Figure 2 shows an example of the wideband ambiguity plane computed for one LFM (Linear Frequency Modulation) received signal.

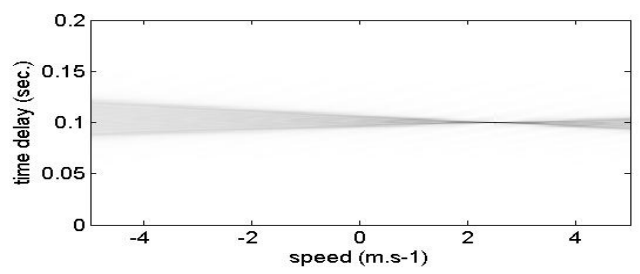

Fig2. Ambiguity function of aLFM: Central frequency $=1300 \mathrm{~Hz}$,

Bandwidth $=2000 \mathrm{~Hz}$, Duration $=4 \mathrm{sec}$. Transmitter speed is $2.5 \mathrm{~m} \cdot \mathrm{s}^{-1}$ and the time delay is 0.1 seconds.

In the multipath case, paths have different apparent speeds and different time delays as illustrated in figure 3. Each path is seen as a sweep-like shape which broadens with the "distance" between the reference and the simulated speed and time-delay.

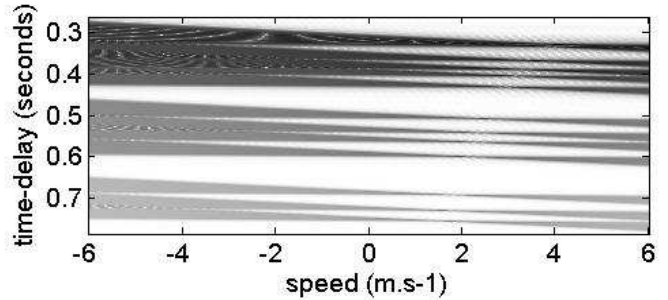

Fig3. Ambiguity plane of a simulated multipath propagation. The relative speed $=8$ knots and the distance source-receiver is $500 \mathrm{~m}$

\section{DOPPLER COMPENSATION TECHNIQUE}

In previous section, the wideband ambiguity function has been introduced in the context of multipath propagation whenever the source is moving or not. A well known method of estimating the IR of one canal of propagation is to compute the cross correlation between a set of time-delayed version of the transmitted signal and the received one. In [1], this method has been shown to be equivalent to taking the values at a speed equal to zero for all the time-delays in the ambiguity plane and it has been shown to give a poor IR because the motion is not taken into account. In [1], the uniform speed compensation, which enables recovering a motionless IR from the ambiguity plane with a moving source, has been introduced. The uniform speed compensation is defined as keeping the column at constant speed $V$ in the wideband ambiguity plane with the hypothesis of a source faraway from the receiver so that the apparent speed of each ray $\left(v_{i}\right)$ will be close to the one of the first path.
The speed $V$ is estimated as the coordinates of the absolute maximum in the ambiguity plane and corresponds to the apparent speed of the first path.

In this paper, we introduce a new Doppler compensation technique estimating the apparent speed of each ray. The principle of the method is to be able detecting the maxima corresponding to each path in the broadband ambiguity function so the motionless IR can be recovered. The difficulty is that paths are very close and interferences creating local maxima appear in the ambiguity function. If one path is removed before computing the ambiguity function then it cannot create any interference with another path and the next arrival should be detectable. The general process of the motion compensation is explained by a scheme presented on figure 4 .

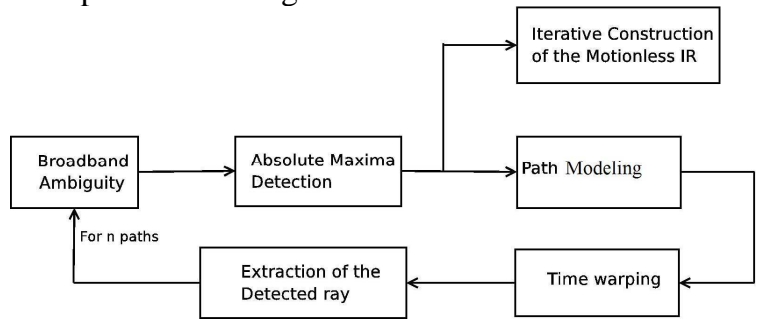

Fig4. Motion compensation using the broadband ambiguity function and time-warping filtering operators.

First, the broadband ambiguity function is computed and the first path is detected as the coordinates of the absolute maximum leading to the apparent speed and the time delay of the first ray. At this point, the first ray of the motionless IR is recovered. The signal received for the first ray is then analytically modeled with formula (6). The next steps consist in removing the detected ray and this cannot be done with classical filtering as paths are too close one from another. The advantage of the time-warping [4] operation is that the detected path can be represented as a pure sine function which can be easily filtered without modifying the received signal. With its analytical expression and timewarping operation, the detected path is removed from the studied record and the inverse time-warping operation is then applied. This leads to a signal containing all paths except the one previously detected. This process is applied $n$ times if one wants to recover an IR with $n$ rays.

\section{RESULTS}

For this simulation, we consider a source moving at $5 \mathrm{~m} \cdot \mathrm{s}^{-1}$, at a depth of 32 meters and at a range of 500 meters from a hydrophone located at 90 meters depth. The channel depth is 165 meters. The signal transmitted is a LFM with a central frequency of $1300 \mathrm{~Hz}$, a bandwidth of $2000 \mathrm{~Hz}$ and a time duration of 4 seconds. 
The figure 5 illustrates the first steps of the method introduced in previous section. The absolute maxima detection is represented with the star in the broadband ambiguity plane (left part of fig. 5). We remark also that the two first paths are really close in the ambiguity plane which creates interferences. Once the absolute maximum detected, the received signal is warped according to the instantaneous frequency law defined by (6). The right part of the figure 5 represents the time-warped space representation where the first path is a pure sine. The signal received for the first ray is extracted with the band-pass filter defined by the two dashed lines represented on figure 5 . This process allows to detect the time delay and amplitude corresponding to the first path and to remove it accurately in order to examine iteratively the other rays. The signals obtained after the extraction of the first ray are shown in the ambiguity plane on figure 6 . It can be seen that the first path is removed without modifying other parts of the signal and the detection of the second ray becomes easier.
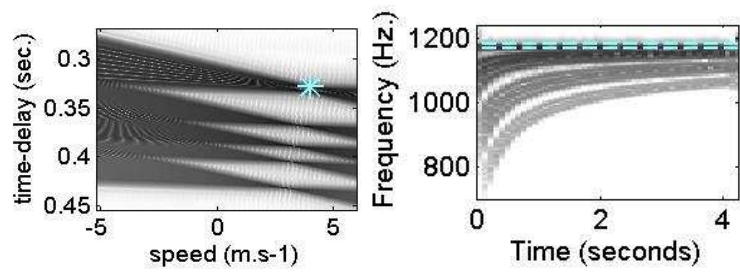

Fig5. Representations of the ambiguity function (left side) and of the time-warped space (right side).
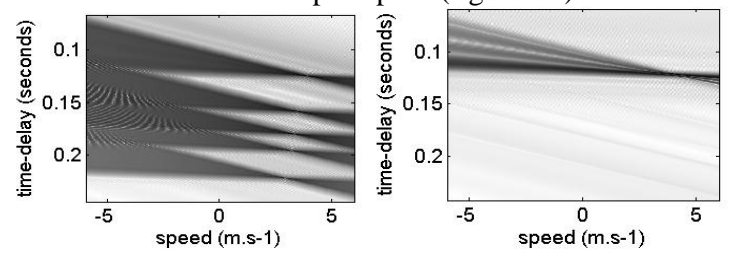

Fig6. Ambiguity functions of the received signal after extracting the first path (left side) and of the signal extracted from the first path.

The results obtained on the simulated data are presented in figure 7 . The picture on the top represents the ambiguity plane of the received signal where the detected local maxima are represented with circles and the theoretical positions (obtained from theoretical results) - with stars. It can be seen that the estimations matches the theory. The solid line represented on the ambiguity plane is the line along which the values of the ambiguity function are kept for the estimation of the motionless IR with the new adaptive speed compensation method. The values of the ambiguity function are kept along the clear dashed line for the uniform speed compensation method and along the dashed line for the uniform speed compensation method. The picture on the bottom illustrates the estimations of the motionless IR zoomed around ray fifth to eighth. The crosses represent the theoretical IR obtained from a motionless source while the curves stand for the different estimation methods. The zero speed compensation is represented with the clear dotted line, the uniform speed compensation with a dashed line and the adaptive speed compensation with a solid line. As expected, the zero speed compensation leads to poor results while motion is not taken into account. The uniform speed compensation leads to a good estimation of the two first rays but the amplitude and the time-delays obtained are biased for other one. Finally, the adaptive speed compensation proposed in this paper allows recovering a motionless IR very close to the theoretic one.
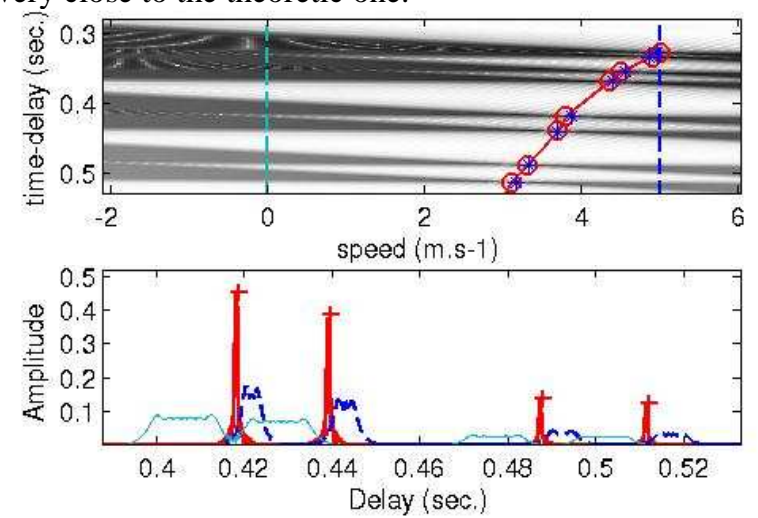

Fig7. Estimation of the motionless IR obtained from different methods in the ambiguity plane and in the time domain.

\section{CONCLUSIONS}

This paper introduces a new method which allows recovering the motionless IR from an unknown constant motion of the transmitter-channel-receiver configuration. The method is based on the warping-based lag-Doppler filtering of arrived signals. This new method can be used for active and passive tomography when the speed of the source is not well monitored and unknown, respectively.

Acknowledgments. This work is supported by research SHOM under grant $\mathrm{N}^{\circ}$ 07CR001.

\section{REFERENCES}

[1] N. Josso, C. Ioana, C. Gervaise, J.I. Mars, "On the Consideration of Motion Effects in Underwater Geoacousitc inversion," J. Acoust. Soc. Am., vol. 123, issue 5, pp. 3625, 2008. [2] F.B. Jensen, W.A. Kuperman, M.B. Porter, H. Schmidt Computational Ocean Acoustics, Ed. New York, Springer-Verlag, 2000.

[3] J.P. Hermand and W.I. Roderick, "Delay-doppler resolution performance of large time-bandwidth product linear FM signals in a multipath ocean environment", J. Acoust. Soc. Am., vol. 84, pp.1709-1727, Nov. 1988.

[4] A. Jarrot, C. Ioana, A. Quinquis, "An extension of the class of unitary time-warping projectors to discrete-time sequences", IEEE ICASSP 2006, Vol. 3, pp. 412-415, Toulouse, France, 2006. 